\title{
MAGNETORHEOLOGICAL SELF-POWERED VIBRATION REDUCTION SYSTEM WITH CURRENT CUT-OFF: EXPERIMENTAL INVESTIGATION
}

\author{
Łukasz JASTRZĘBSKI*, Bogdan SAPIŃSKI* \\ *AGH University of Science and Technology, Mechanical Engineering and Robotics, Department of Process Control, \\ Al. Adama Mickiewicza 30, 30-059 Kraków, Poland \\ lukasz.jastrzebski83@gmail.com,deep@agh.edu.pl
}

received 25 January 2018, revised 18 May 2018, accepted 20 May 2018

\begin{abstract}
The paper summarises the results of laboratory testing of an energy harvesting vibration reduction system based on a magnetorheological (MR) damper whose control circuit incorporates a battery of bipolar electrolytic capacitors (current cut-off circuit). It is designed to reduce the undesired effects in vibration reduction systems of this type, associated with the increasing amplitude of the sprung mass vibration under the excitation inputs whose frequency should exceed the resonance frequency of the entire system. Results have demonstrated that incorporating a current cut-off circuit results in a significant decrease of sprung mass vibration amplitudes when the frequency of acting excitation inputs is higher than the resonance frequency.
\end{abstract}

Key words: MR Damper, Vibration, Energy Harvesting, Current Cut-Off Circuit

\section{INTRODUCTION}

One of the first studies on MR-damper based vibration reduction systems with energy harvesting capability was the work (Choi et al., 2007) investigating the performance of an electromagnetic energy harvester converting the energy of vibration into electric power supplying the control coil in an MR damper. The harvester was able to generate sufficiently high energy to power-supply the RD-1097-01 damper (Lord Co) in the analysed system. Further works provide information about other design versions of electromagnetic harvesters, methods of their integration with MR dampers and suggested implementations in vibration reduction systems. For example, Chen and Liao (2012) presents theoretical and experimental studies of an MR damper prototype which had self-powered and self-sensing capabilities. The work (Choi and Werely, 2009) investigates experimentally a smart passive control system comprising an MR damper and an electromagnetic induction device to generate electrical power. The studies: Sapiński, (2008) and Sapiński (2010) concern design considerations, calculations of magnetic field and experimental tests of the electromagnetic induction devices, that could be integrated with an MR damper. The reports (Wang et al., 2009) and (Wang and Bai, 2013) demonstrate the idea of an integrated relative displacement self-sensing MR damper and also designing, fabrication and experimental testing of its prototype. The study (Xinchun et al., 2015) presents a novel self-powered MR damper focusing on its theoretical and experimental analysis. The work (Zhu et al., 2012) describes self-powered and sensor-based MR damper systems and its usefulness in large-scale civil constructions where the power supply is impractical.

It is worthwhile to mention that such harvesters can act not only as power generators, but as velocity sensors as well. A comprehensive review of literature on the subject of energy harvesting and recent advancements in MR dampers is provided in Ahmed et al. (2016).
Research data demonstrate that MR damper-based vibration reduction systems with energy harvesting capability are able to effectively reduce the amplitude of the sprung mass vibration in the neighbourhood of the resonance frequency. However, because the harvester and a MR damper are connected in between the sprung mass and the source of vibration, the amplitude of the sprung mass vibrations will significantly increase when the excitation frequency becomes higher than the resonance frequency of the system (Sapiński, 2011; Sapiński et al., 2011). This undesired effect is attributed to the increased amount of energy generated by the harvester (increasing the relative velocity), hence the amount of energy supplied to power the MR damper control coil has to rise, which in consequence leads to an increase of dissipated energy. This problem can be overcome by incorporating a passive (Jastrzębski and Sapiński, 2016) or controlled electric circuit connected in between the harvester coil and the MR damper control coil (Sapiński et al., 2011; 2016).

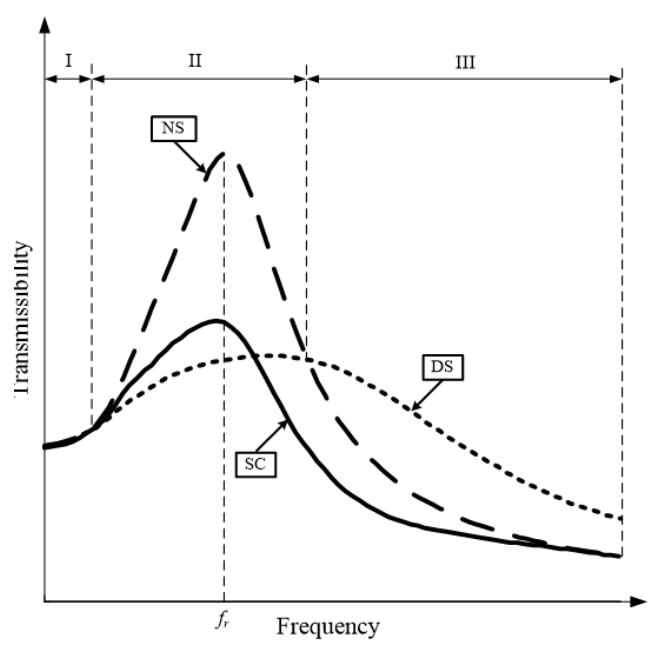

Fig. 1. Frequency response of the vibration reduction system 
Fig. 1 shows vibration transmissibility of a 1 DOF system, for three variants of the damper coil supply type (NS - no power supply, DS - directly supplied from the harvester, SC - supplied from the harvester via a battery of capacitors. Three characteristics frequency ranges are designated as I, II, III.

In the low frequency range I $\left(f<<f_{r}\right)$, vibration amplitudes are similar in all three investigated cases. In the frequency range $\|\left(f \approx f_{r}\right)$ in the DS case the vibration amplitude tends to decrease in relation to that registered in the NS case. In the frequency range III $\left(f>>f_{r}\right)$ the amplitude of vibrations in the case DS increases when compared to NS. This undesired effect is not observed in the SC case.

It is suggested, therefore, that a battery of capacitors comprising a current cut-off circuit should be connected in between the harvester coil and the MR damper coil. Consequently, at frequencies in excess of $f_{r}$, the current in the damper coil will be cut-off, thus enabling the amplitudes of the sprung mass vibrations to be effectively reduced.

\section{CURRENT CUT-OFF CIRCUIT}

Fig. 2 shows the schematic diagram of the electric circuit comprising the harvester coil $\left(R_{h}, L_{h}\right)$, capacitor batteries $\left(C_{1}, \ldots ., C_{n}\right)$ and the damper coil $\left(R_{d}, L_{d}\right)$. It is worthwhile to mention that at frequency $f<<f_{r}$, reactance of the condenser battery $X_{c} \rightarrow \infty$, hence $i_{h} \approx i_{d}$. At frequency $A>f_{r}$, reactance $X_{c} \rightarrow 0$ and hence the current $i_{d} \rightarrow 0$ (ih $\approx i_{c}$ ). Accordingly, the capacitance of the capacitors' battery has to be precisely controlled in relation to the resonance frequency $\mathrm{fr}_{\mathrm{r}}$. Thus, the effects of the capacitors' capacitance on frequency characteristics of the current cut-off circuit and the vibration reduction system are analysed and comparison is made of the system's performance in the NS, DS, SC cases. The battery comprised 2, 4, 6, 8 capacitors connected in parallel, each having the capacitance $4700 \mu \mathrm{F}$ and voltage rating 35 $\mathrm{V}$, the total capacitance of the battery being $9.4 \mathrm{mF}$ (variant SC1), $18.8 \mathrm{mF}$ (variant SC2), $28.2 \mathrm{mF}$ (variant SC3) and $37.6 \mathrm{mF}$ (variant SC4).

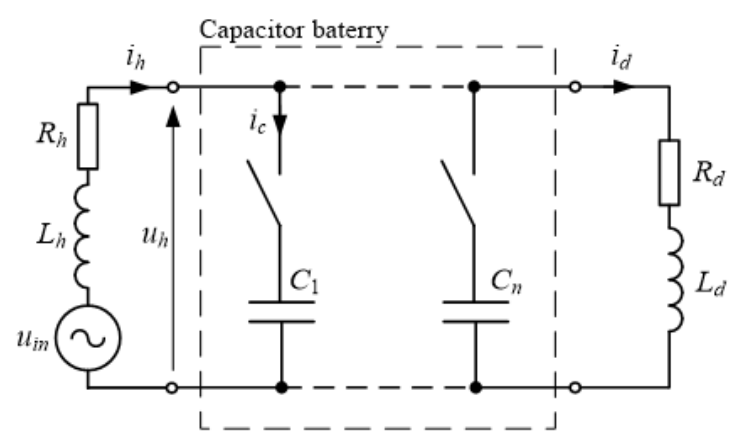

Fig. 2. Schematic of the electric circuit: harvester coil, capacitors, damper coil

In the first stage the relationship was established between the impedance of the system and frequency, and the effectiveness of current cut-off in the damper coil was investigated. The diagram of the measurement system is shown in Fig. 3 The hardware includes a power supply, a power amplifier (based on an operational amplifier OPA549 and integrated circuits for output current and voltage measurements), a PC with an AD/DA card and current-to-voltage converters. The software is supported by the MATLAB/Simulink environment. The excitation signal applied was a sinusoidal current $i_{h}$ with amplitude $0.5 \mathrm{~A}$ and frequency $f$ from the range $0.1-10 \mathrm{~Hz}$, varied with the step $0.1 \mathrm{~Hz}$ (current mode of the amplifier operation).

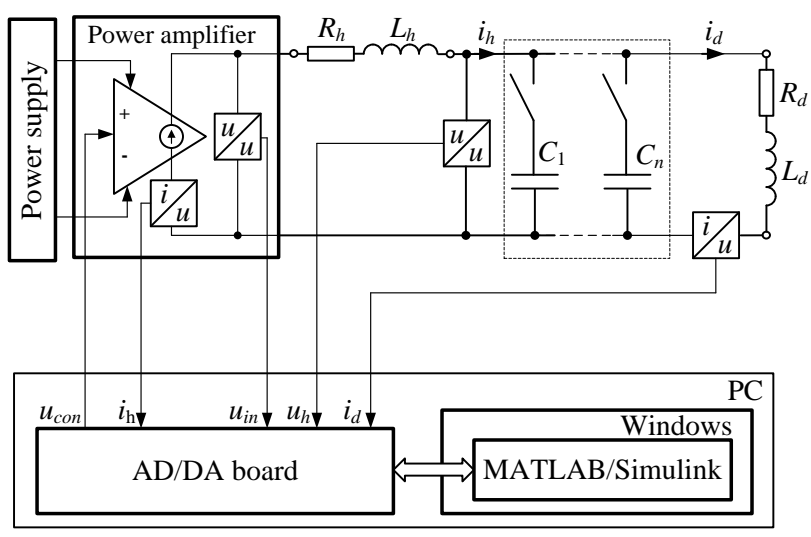

Fig. 3. Diagram of the measurement system for current cut-off circuit

Fig. $4-6$ summarise the test results presented as impedance vs frequency plots. The modulus of total impedance of the system $\left|Z_{c}\right|$ (see Fig. 2) is derived from formulas (1), likewise the impedance $|Z|$ is expressed as $U_{h} / / h$.

$$
\left|Z_{c}\right|=\frac{U_{\text {in }}}{I_{h}}=\frac{\sqrt{\frac{1}{T} \int_{t}^{t+T} u_{i n}(t)^{2} d t}}{\sqrt{\frac{1}{T} \int_{t}^{t+T} i_{h}(t)^{2} d t}}
$$

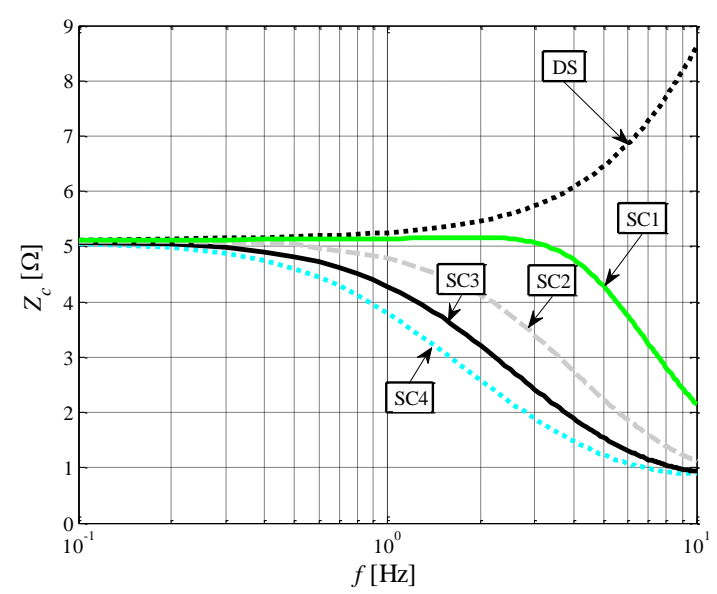

Fig. 4. Module of total impedance $\left|Z_{c}\right|$ vs. frequency $f$

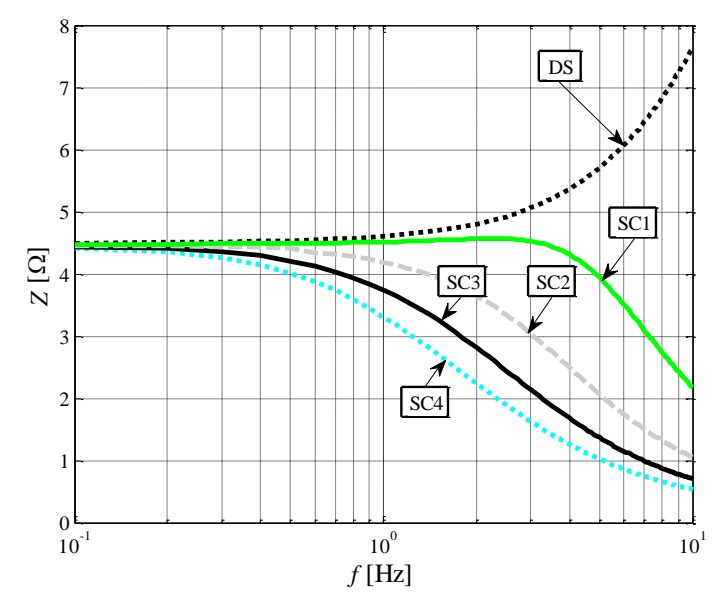

Fig. 5. Module of impedance $|Z|$ vs. frequency $f$ 


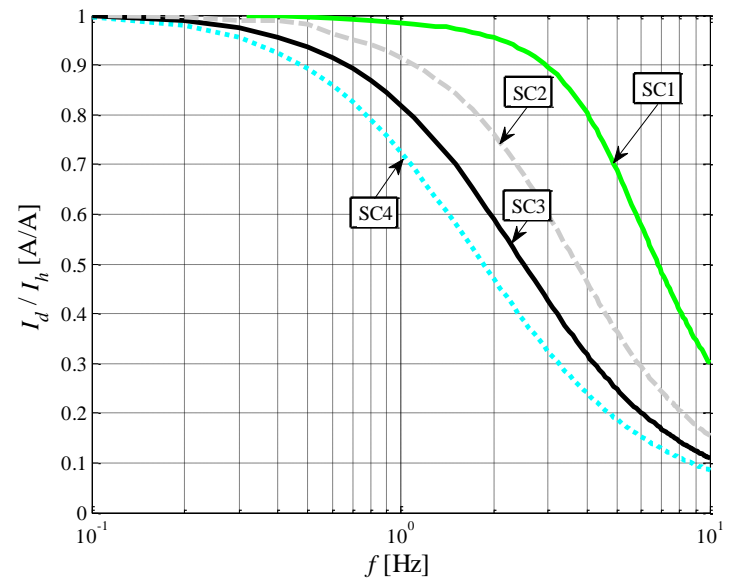

Fig. 6. Current ratio $l_{d} / l_{h}$ vs. frequency $f$

It appears that at frequency $f=0.1 \mathrm{~Hz}$, the difference between $\left|Z_{c}\right|$ and $|Z|$ is equal to the internal impedance of the harvester, which is $0.65 \Omega$ (compare Figs. 4 and 5 ). In the case $D S$, an increase in frequency gives rise to increase of both $\left|Z_{c}\right|$ and $|Z|$, which is revealed when $f>1 \mathrm{~Hz}$ (the circuit behaviour becomes resistive and inductive). In the case SC a reverse effect is observed, i.e. $\left|Z_{c}\right|$ and $|Z|$ tend to decrease whilst the circuit behaves like a resistive-capacitive one. The impedance modulus $\left|Z_{c}\right|$ decreases $\sqrt{2}$ - fold, respectively at $f>6.4 \mathrm{~Hz}$ (SC1), $f>2.8$ $\mathrm{Hz}(\mathrm{SC} 2), f>1.7 \mathrm{~Hz}(\mathrm{SC} 3)$ and $f>1.2 \mathrm{~Hz}$. At frequency $f=0.1 \mathrm{~Hz}$, $I_{h}=I_{d}$, due to a large impedance of capacitors (see Fig. 6). When frequency $f$ increases, the current in capacitors increases too because their impedance will go down, causing the current $I_{d}$ in the damper coil to decrease (current cut-off). Current $l_{d}$ is $\sqrt{2}$ - fold lower than $\ln$ at frequencies $f>4.8 \mathrm{~Hz}(\mathrm{SC} 1), f>2.3 \mathrm{~Hz}$ (SC2), $f>1.5 \mathrm{~Hz}(\mathrm{SC} 3), f>1.1 \mathrm{~Hz}$ (SC4).

\section{MR SELF-POWERED VIBRATION REDUCTION SYSTEM WITH CURRENT CUT-OFF}

The current cut-off circuit outlined in the previous section has been implemented in a MR damper-based vibration reduction system with energy harvesting capability (1 DOF system). Tests were performed to investigate how the capacitors' capacitance should affect the frequency characteristics of the system. Thus obtained results were compared with those obtained when investigating NS and DS cases.

The experimental set-up (Fig. 7) described in more detail in Sapiński et al. (2010) consisted of the electro-dynamic shaker (model V780 manufactured by the LDS Company), a mobile platform (sprung mass) with the mass $100 \mathrm{~kg}$, a vibration reduction system (electromagnetic harvester (Sapiński, 2010) and a Lord Co. RD-1005-3 series MR damper), a spring with the stiffness coefficient $k=105 \mathrm{~N} / \mathrm{m}$ and sensors $S_{1}-S_{3}$. The sprung mass, damper, a harvester and springs comprise a 1 DOF system whose natural frequency equals $f_{0}=5 \mathrm{~Hz}$. Displacements of the shaker core $z(t)$ and of the platform $x(t)$ were measured with laser sensors $\left(S_{1}, S_{2}\right)$. Measurements of the damper force $F_{d}\left(S_{3}\right)$ were taken with a force sensor. Current levels in the harvester coil in and in the damper coil $i_{d}$ were measured using current-to-voltage converters.

Experiments were conducted under the applied sine excitations $z(t)$ with the amplitude $3.5 \mathrm{~mm}$ and frequency varied in the range
2 - $10 \mathrm{~Hz}$, with the step $0.1 \mathrm{~Hz}$. Measurements signals were registered within the time period $20 \mathrm{~s}$, the sampling frequency being $1 \mathrm{kHz}$.

Figs 8-13 show the frequency characteristics obtained in the NS, DS, SC cases. The displacement transmissibility coefficient $T_{x z}$ is derived from formula (2). Frequency characteristics provided in further sections involve the rms values of investigated quantities.

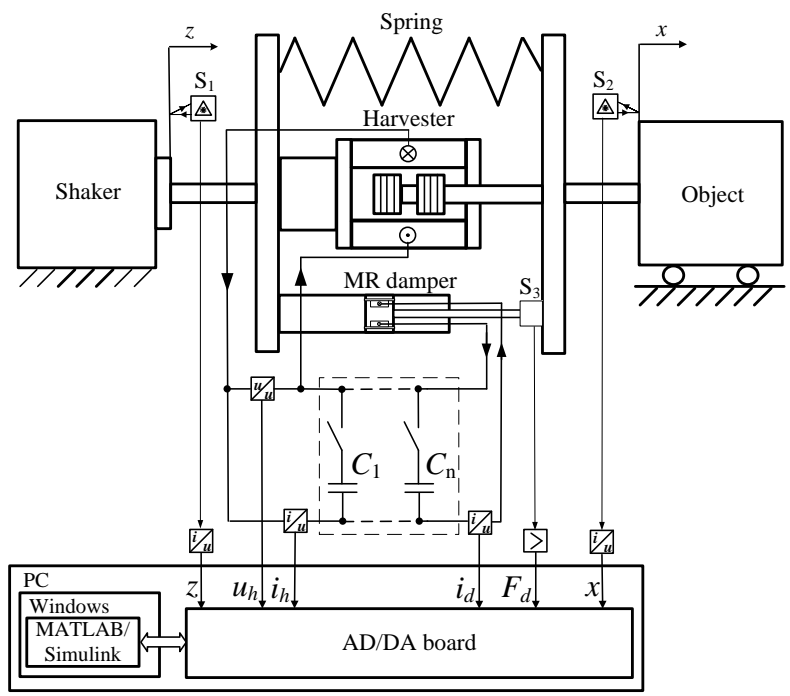

Fig. 7. Schematic diagram of the experimental set-up

$T_{x Z}=\frac{X}{Z}=\frac{\sqrt{\frac{1}{T} \int_{t}^{t+T} x(t)^{2} d t}}{\sqrt{\frac{1}{T} \int_{t}^{t+T} z(t)^{2} d t}}$

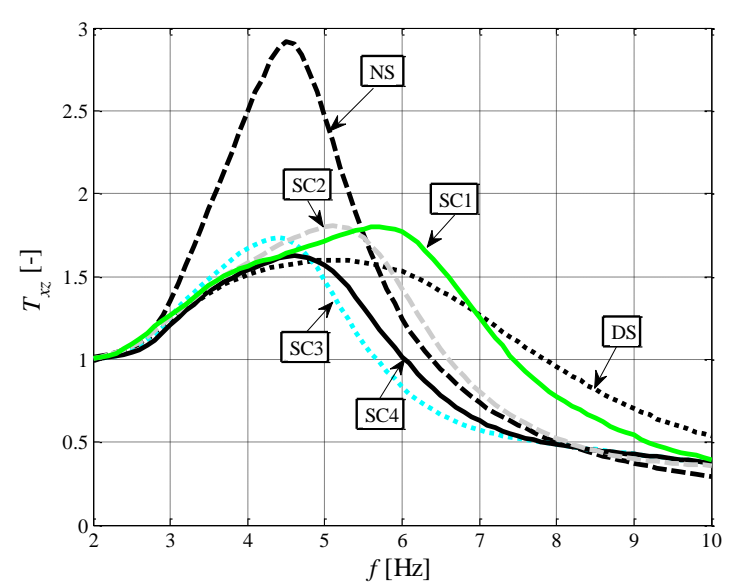

Fig. 8. Transmissibility coefficient $T_{x z}$ vs. frequency $f$

The maximal value of $T_{x z}$ (Fig. 8) is 2.92 (NS case) and 1.6 (DS case), obtained at respective frequencies $f=4.5 \mathrm{~Hz}$ and $f=5$ $\mathrm{Hz}$. It appears that the maximal amplitude of vibration registered in the DS case is nearly 1.8-fold lower than in the NS case. Interestingly, at frequency $f=10 \mathrm{~Hz}$, the amplitude of vibrations in the DS case is found to be 1.8-fold higher than in the NS case.

In the frequency range $(2,8) \mathrm{Hz}$ in which the values of $T_{x z}$ tend to decrease in relation to the NS case, the variants SC3 and SC4 appear to be most favourable. Further, in the entire frequency range the amplitude of vibrations in the variant SC4 was lower 
when compared to DS. At frequency $f=10 \mathrm{~Hz}, T_{x z}$ in the SC case was found to be 1.4-fold lower in relation to DS case and 1.3-fold higher than in the NS case.

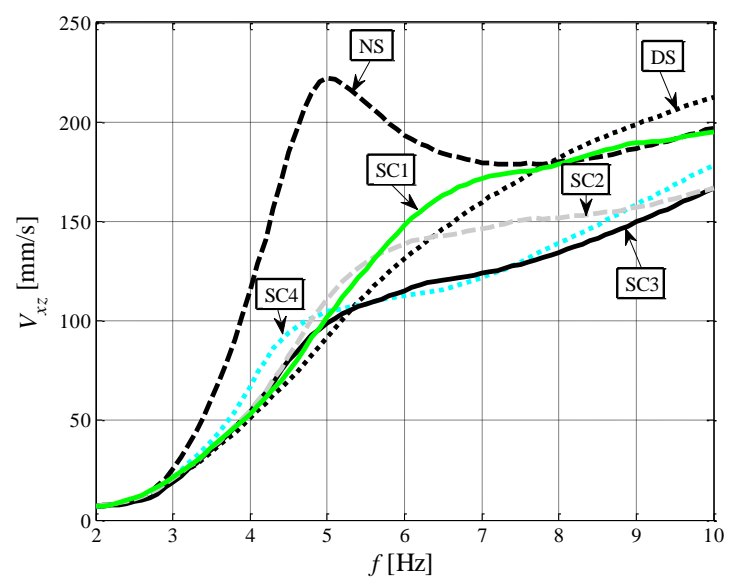

Fig. 9. Relative velocity $V_{x z}$ vs. frequency $f$

The highest rms values of relative velocity $V_{x z}$ are registered in the case NS, in the frequency range $(3,8) \mathrm{Hz}$, and in the DS case at frequencies $f>8 \mathrm{~Hz}$ (see Fig. 9). In the frequency range $(2,3) \mathrm{Hz}$ the values of $V_{x z}$ registered in the cases NS, DS, SC are found to be similar.

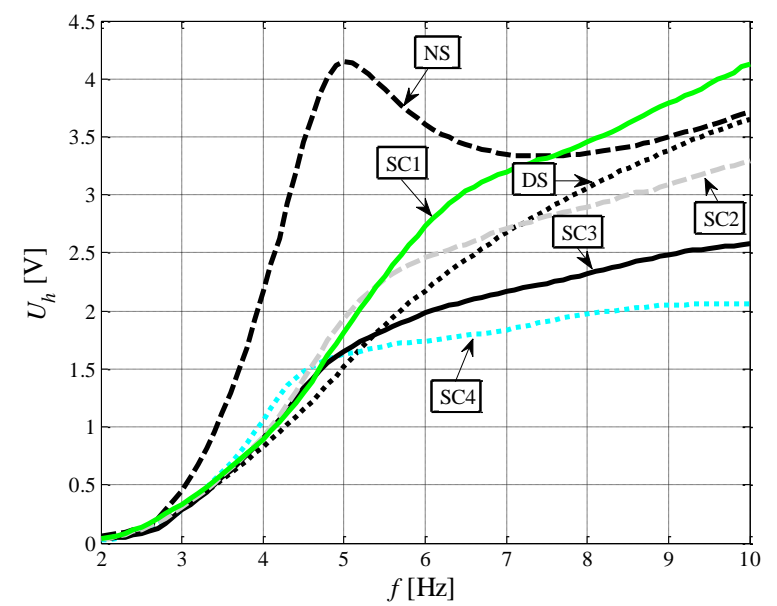

Fig. 10. RMS harvester voltage $U_{h}$ vs. frequency $f$

When comparing plots in Figs. 9 and 10, it is apparent that the rms value of $U_{h}$ in the NS case is proportional to relative velocity $V_{x z}$, reaching its maximal value at frequency $f=5 \mathrm{~Hz}$. In the $D S$ and SC cases, voltage $U_{h}$ tends to increase with frequency $f$. It appears that for the same velocity $V_{x z}$, the voltage $U_{h}$ registered in the variant SC4 is lower than in the variant SC3, which is attributable to higher currents $I_{h}$ in the circuit.

It is readily apparent (see plots in Fig. 11) that rms value of current $l_{h}$ tends to increase with increasing capacitance of the condenser battery. At frequency $f=10 \mathrm{~Hz}$, the current output $l_{h}$ in the variant SC is 5 times as high as that registered in the variant SC1 and that registered in the variant SC4 is 9 times higher than in the DS case.

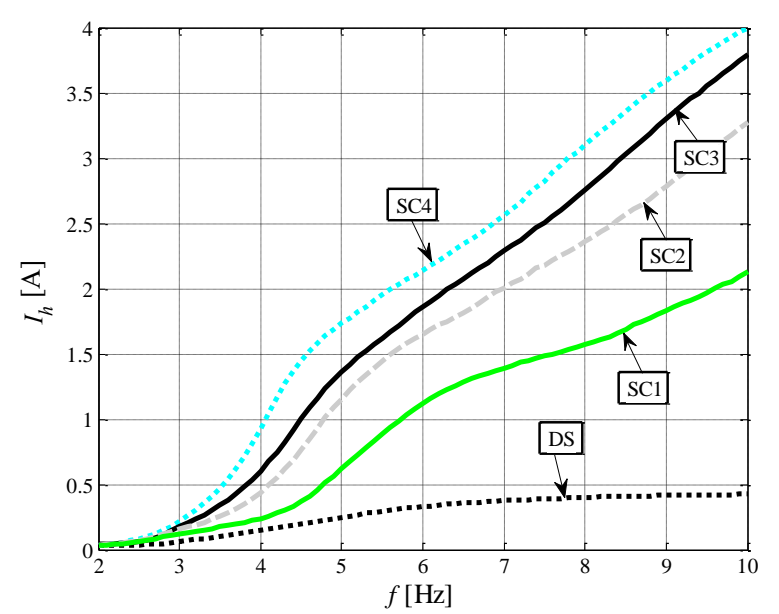

Fig. 11. RMS harvester current $\ln$ vs. frequency $f$

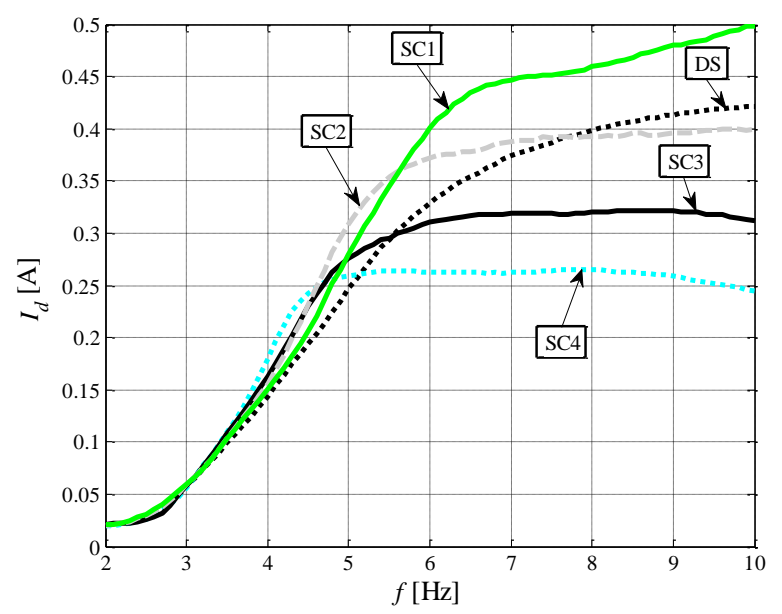

Fig. 12. RMS damper current $l_{d}$ Vs. frequency $f$

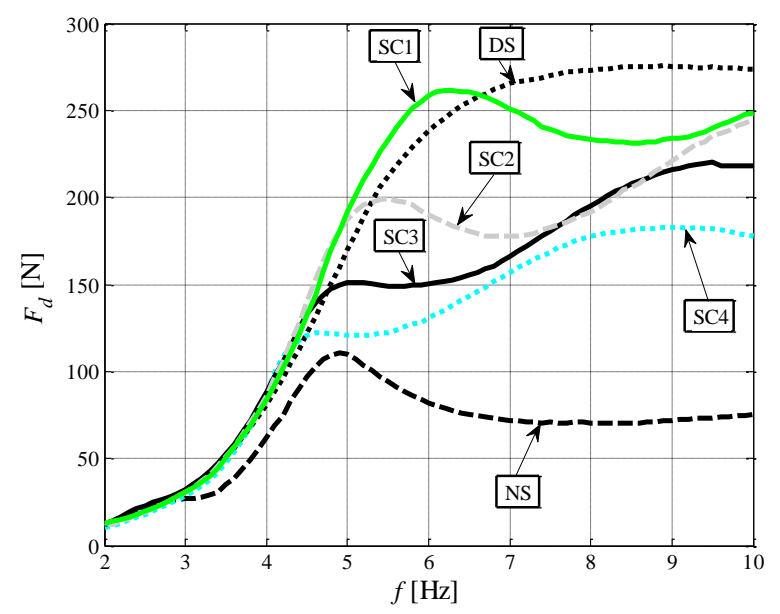

Fig. 13. RMS damper force $F_{d}$ vs. frequency $f$

In the DS case an increase in frequency $f$ results in an increase of the current rms value $l_{d}$ (Fig. 12). In the variants SC2, SC3, SC4 no significant increase of the current $l_{d}$ is registered at frequencies $f>6 \mathrm{~Hz}$, which is attributed to a slower rate of voltage increase $U_{h}$ (see Fig. 10) and a higher reactance $X_{c}$ of the condenser battery. Actually, the higher the capacitance of the condenser battery, the lower current $l_{d}$ at the frequency $f>6 \mathrm{~Hz}$.

The lowest rms values of the damping force $F_{d}$ (Fig. 13) are registered in the NS case. The maximal value of the force $F_{d}$ is 
attained at the frequency $f=4.9 \mathrm{~Hz}$, coinciding with the maximal velocity $V_{x z}$ (see Fig. 9). In the case DS, force $F_{d}$ tends to increase with frequencies, at frequencies $f<8 \mathrm{~Hz}$. For frequencies in excess of $8 \mathrm{~Hz}$, the force $F_{d}$ remains constant. In the SC case, at frequencies $f>7 \mathrm{~Hz}$, the force $F_{d}$ has lower values when compared to the DS case, which can be attributed to decrease in current $l_{d}$ (see Fig. 12) and velocity $V_{x z}$ (see Fig. 9).

\section{SUMMARY}

Investigations of a MR damper-based vibration reduction system with energy harvesting capability clearly demonstrate that at frequencies in excess of the resonance frequency, the increase of the vibration amplitude can be limited through the use of a current cut-off circuit (consisting of a battery of bipolar electrolytic capacitors). Results show that the system performance in the variant SC3 and SC4 is most satisfactory in terms of effective reduction of the sprung mass vibration amplitudes. Comparison of vibration amplitudes registered in those two variants and in the DS case reveals their decrease in the entire frequency range. As regards the NS case, however, amplitudes tend to decrease in the frequency range $(2,8) \mathrm{Hz}$ only.

In particular, at frequency $10 \mathrm{~Hz}$ the vibrations amplitude in the variants SC3 and SC4 is higher by $30 \%$ than that registered in the NS case, whilst in the DS case, there is an amplitude increase by nearly $80 \%$.

Further research efforts will focus on investigations of passive circuits utilising the voltage resonance effects, in order to improve the overall performance of a MR damper-based vibration reduction system with energy harvesting capability.

\section{REFERENCES}

1. Ahamed R., Ferdaus Md. M., Li Y. (2016), Advancement in energy harvesting magneto-rheological fluid damper: A review, KoreaAustralia Rheology Journal, 28(4), 355-379.

2. Chen C., Liao W.H. (2012), A self-sensing magnetorheological damper with power generation, Smart Materials and Structures, 21, 025014.

3. Choi K.M., Jung H. J., Lee H. J, Cho S.W. (2007), Feasibility study of smart passive control system equipped with electromagnetic induction device, Smart Materials and Structures, 16, 2323-2329.

4. Choi Y.T., Werely N.M (2009), Self-powered magnetorhelogical dampers, Journal of Vibration and Acoustics, 131, 44-50.

5. Jastrzębski Ł., Sapiński B. (2016), Electrical interface for a selfpowered MR damper-based vibration reduction system, Acta Mechanica et Automatica, 10(3), 165-172.

6. Sapiński B., Snamina J., Jastrzębski Ł., Staśkiewicz A., (2011), Laboratory stand for testing of self-powered vibration reduction systems, Journal of Theoretical and Applied Mechanics, 49(4), 11691181.

7. Sapiński B. (2008), An experimental electromagnetic induction device for a magnetorheological damper, Journal of Theoretical and Applied Mechanics, 46(4), 933-947.

8. Sapiński B. (2010), Vibration power generator for a linear MR damper, Smart Materials and Structures, 19, 105012.

9. Sapiński B. (2011), Experimental study of a self-powered and sensing MR damper-based vibration control system, Smart Materials and Structures, 20, 105007.

10. Sapiński B., Rosół M., Jastrzębski Ł. (2011), Charakterystyki semiaktywnego układu redukcji drgań z odzyskiem energii, Pomiary, Automatyka, Kontrola, 57(5), 502-506.

11. Sapiński B., Rosół M., Węgrzynowski M. (2016), Investigation of an energy harvesting MR damper in a vibration control system, Smart Materials and Structures, 25, 125017.

12. Wang D.H., Bai X.X. (2013), A magnetorheological damper with an integrated self-powered displacement sensor, Smart Materials and Structures, 22, 075001

13. Wang D.H., Bai X.X., Liao W.H. (2009), Principle, design and modeling of an integrated relative displacement magnetorheological damper based on electromagnetic induction, Smart Materials and Structures, 18, 095025.

14. Xinchun G., Yonghu H., Yi R., Hui L., Jinping O. (2015), A novel self-powered MR damper: Theoretical and experimental analysis, Smart Materials and Structures, 24, 105033.

15. Zhu S.Y., Shen W.A., Xu Y.L., Lee W.C. (2012), Linear electromagnetic devices for vibration damping and energy harvesting: Modeling and testing, Engineering Structures, 34, 198-212.

This work is supported by AGH University of Science and Technology under research program No. 15.11.130.431. 